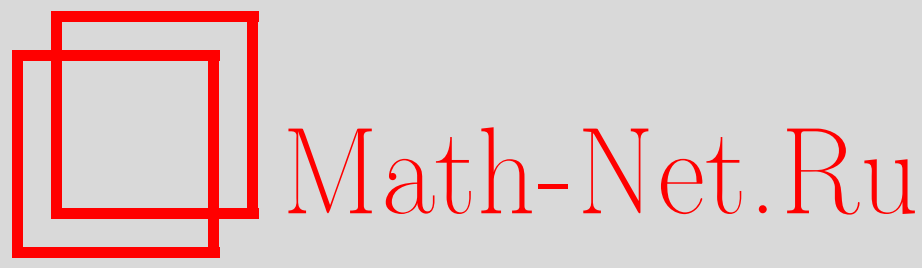

3. Х. Закирова, О структуре проективной группы в псевдоримановом пространстве, ТМФ, 2018, том 196, номep 1, 30-41

DOI: https://doi.org/10.4213/tmf9492

Использование Общероссийского математического портала Math-Net.Ru подразумевает, что вы прочитали и согласны с пользовательским соглашением http://www.mathnet.ru/rus/agreement

Параметры загрузки:

IP : 54.237 .59 .107

26 апреля 2023 г., 18:34:34

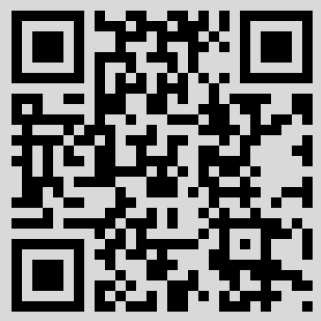




\title{
ФИЗИКА
}

Том 196, № 1

июль, 2018

(C) 2018 г.

\section{О СТРУКТУРЕ ПРОЕКТИВНОЙ ГРУППЫ В ПСЕВДОРИМАНОВОМ ПРОСТРАНСТВЕ}

\begin{abstract}
Проведено исследование $n$-мерных псевдоримановых пространств $V^{n}\left(g_{i j}\right)$ с произвольной сигнатурой, которые допускают проективные движения, т. е. группы непрерывных преобразований, сохраняющих геодезические. В частности, найдена метрика псевдориманова пространства специального типа и установлены важные проективно-групповые свойства этого пространства.
\end{abstract}

Ключевые слова: дифференциальная геометрия, общая теория относительности, псевдоримановы многообразия, системы дифференциальных уравнений с частными производными.

DOI: https://doi.org/10.4213/tmf9492

\section{1. ВВЕДЕНИЕ}

Преобразование $f$ псевдориманова многообразия $M$ на себя называется $n$ роективным преобразованием, если оно переводит геодезические линии в геодезические линии.

Линия $x^{i}(t)$ называется геодезической, если ее вектор скорости $T^{i}=d x^{i} / d t$ остается параллельным самому себе при переносе вдоль кривой (см. книгу [1]): $\nabla_{t} T=0$. Уравнение геодезических в локальных координатах имеет вид

$$
\frac{d^{2} x^{i}}{d t^{2}}+\Gamma_{j k}^{i} \frac{d x^{j}}{d t} \frac{d x^{k}}{d t}=0
$$

где $\Gamma_{j k}^{i}$ - компоненты связности псевдориманова многообразия $(M, g)$. Отметим, что здесь и далее по повторяющимся индексам идет суммирование.

Векторное поле $X$ называется инфинитезимальным проективным преобразованием или проективным движением, если локальная однопараметрическая группа преобразований, порождаемая этим полем в окрестности каждой точки $p \in M$, состоит из локальных проективных преобразований.

Работа выполнена при финансовой поддержке РФФИ (грант № 16-01-00291_а).

* Казанский государственный энергетический университет, Казань, Россия. E-mail: zolya_zakirova@mail.ru 
Векторное поле $X$ является инфинитезимальным проективным преобразованием на многообразии $M$ с аффинной связностью $\nabla$ тогда и только тогда, когда [2]

$$
\nabla_{Y}\left(L_{X} Z-\nabla_{X} Z\right)-\left(L_{X}-\nabla_{X}\right) \nabla_{Y} Z=R(X, Y) Z-\varphi(Y) Z-Y \varphi(Z)
$$

для поля 1-формы $\varphi$ и всех векторных полей $Y, Z$ на $M$, где $R$ - тензор кривизны.

Если $M$ - псевдориманово многообразие с метрикой $g$ и римановой связностью $\nabla$, то условие (1) эквивалентно уравнениям (см. работу [2])

$$
\begin{gathered}
L_{X} g=h, \\
\nabla h(Y, Z, W)=2 g(Y, Z) W \varphi+g(Y, W) Z \varphi+g(Z, W) Y \varphi,
\end{gathered}
$$

где $(Y, Z, W) \in T(M), \varphi=\operatorname{div} X /(n+1)$. Уравнение $(2)$ называется обобщенным уравнением Киллинга, уравнение (3) называется уравнением Эйзенхарта.

В последние годы значительно возрос интерес к геометрическим свойствам многомерных пространств с нестандартными сигнатурами. В основном он связан с теорией струн, где временное(ые) направление(ия) в пространстве-времени связано(ы) с нестандартным знаком кинетического члена в действии лиувиллевского(их) поля(ей). Множество работ посвящено исследованию многомерных космологических моделей. Цель этих работ - установить, насколько далеко можно продвинуться в объяснении свойств четырехмерной Вселенной с помощью геометрических величин дополнительных измерений. Стоит отметить, что пространственно-временные симметрии порождают законы сохранения энергии, импульса и момента импульса. В частности, инфинитезимальные проективные и аффинные преобразования приводят к фундаментальным полевым и механическим законам сохранения в форме квадратичных первых интегралов уравнений геодезических.

Впервые проблема определения двумерных римановых многообразий, которые допускают проективные движения или инфинитезимальные проективные преобразования, т. е. непрерывные группы преобразований, сохраняющих геодезические, рассматривались Ли и Кенигсом (см. книгу [3]). Другие важные результаты были получены Петровым в работе [4], где он классифицировал геодезически эквивалентные псевдоримановы пространства $V^{3}$. Для риманова многообразия с размерностью, большей двух, похожая проблема была решена Фубини в статье [5] и Солодовниковым в работе [6].

В работе [7] Аминова классифицировала все лоренцевы многообразия размерности не меньше трех, допускающие негомотетические инфинитезимальные проективные и аффинные преобразования. В каждом случае были определены соответствующие максимальные проективные и аффинные алгебры Ли. Автором настоящей статьи в кандидатской диссертации [8] были исследованы шестимерные псевдоримановы пространства с сигнатурой $[++----]$. Частично результаты были опубликованы в работах [9]-[13]. Данная проблема не решена для $n$-мерного псевдориманова пространства с произвольной сигнатурой.

Для того чтобы найти псевдориманово пространство, допускающее негомотетическое инфинитезимальное проективное преобразование, нужно проинтегрировать уравнение Эйзенхарта (3). Задача определения таких пространств зависит от типа $h$-пространств, т. е. от типа билинейной формы $L_{X} g$, которая определяется характеристикой Сегре $\lambda$-матрицы $h-\lambda g$ (см. статью [7]). Если характеристика тензора $L_{X} g$ 
есть $[a b c . .$.$] , то мы будем называть соответствующее пространство h$-пространством типа $[a b c \ldots]$. Таким образом, псевдориманово пространство, для которого существует нетривиальное решение $h \neq c g$ уравнения Эйзенхарта, называется $h$-npocmранством.

Число возможных типов зависит от размерности и сигнатуры псевдориманова пространства. В частности, в случае лоренцевых многообразий возможны $h$-про-

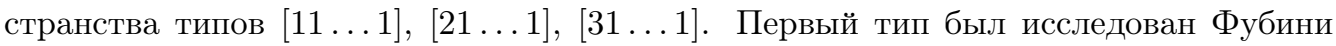
в работе [5] и Солодовниковым в статье [6], другие два были исследованы Аминовой в работе [7]. Целью настоящей работы является исследование $n$-мерного псевдориманова пространства $V^{n}\left(g_{i j}\right)$ типа [41 ...1].

Основной метод определения псевдоримановых многообразий, допускающих негомотетическую проективную группу $G_{r}$, был развит Аминовой (см. статью [7]). Используя в настоящей работе технику интегрирования в косонормальном (подвижном) репере, мы находим метрику рассматриваемого $h$-пространства.

Уравнение Эйзенхарта в косонормальном репере имеет вид [7]

$$
X_{r} \bar{a}_{p q}+\sum_{h=1}^{n} e_{h}\left(\bar{a}_{h q} \gamma_{\tilde{h} p r}+\bar{a}_{p h} \gamma_{\tilde{h} q r}\right)=\bar{g}_{p r} X_{q} \varphi+\bar{g}_{q r} X_{p} \varphi, \quad p, q, r=1, \ldots, n,
$$

где

$$
X_{r} \varphi \equiv \xi_{r}^{i} \frac{\partial \varphi}{\partial x^{i}}, \quad \gamma_{p q r}=-\gamma_{q p r}=\xi_{p} \xi_{q} \underset{r}{\xi_{r}^{i}} \xi_{r}^{j}, \quad a_{i j}=h_{i j}-2 \varphi g_{i j},
$$

$\xi_{i}^{j}$ - компоненты косонормального репера, $\bar{g}_{p r}=e_{p} \delta_{\tilde{p}}^{r}$ и $\bar{a}_{p q}-$ канонические формы тензоров $g_{p r}, a_{p q}$ соответственно, $\gamma_{l k}^{p}=e_{p} \gamma_{l \tilde{p} k}$ - компоненты связности в косонормальном репере $X$.

Коммутаторы векторных полей $X_{k}$ и $X_{h}$ определяются по формуле [7]

$$
\left[X_{k}, X_{h}\right]=\sum_{l=1}^{n} e_{l}\left(\gamma_{l k h}-\gamma_{l h k}\right) X_{\tilde{l}}
$$

Отображение $\sim$, которое переводит одни индексы в другие, было введено в определении косонормального репера. Опуская громоздкое определение косонормального (подвижного) репера, достаточно привести несколько примеров, и читателям станет понятно действие отображения . Например, для $h$-пространства типа [221 . . 1] $\tilde{1}=2, \tilde{2}=1, \tilde{3}=4, \tilde{4}=3, \tilde{5}=5, \tilde{6}=6$ и т. д.; для $h$-пространства типа [321 ... 1$]$ $\tilde{1}=3, \tilde{2}=2, \tilde{3}=1, \tilde{4}=5, \tilde{5}=4, \tilde{6}=6$ и т. д. Для $h$-пространства типа [41 ... 1] $\tilde{1}=4, \tilde{2}=3, \tilde{3}=2, \tilde{4}=1, \tilde{5}=5, \tilde{6}=6$ и т. д.

Для $n$-мерного $h$-пространства типа $[41 \ldots 1]$ канонические формы $\bar{g}_{p r}$ и $\bar{a}_{p q}$ имеют вид (см. работу [4])

$$
\begin{aligned}
& \bar{g}_{i j} d x^{i} d x^{j}=e\left(2 d x^{1} d x^{4}+2 d x^{2} d x^{3}\right)+\sum_{\sigma=5}^{n} e_{\sigma} d x^{\sigma^{2}}, \\
& \bar{a}_{i j} d x^{i} d x^{j}=e \lambda\left(2 d x^{1} d x^{4}+2 d x^{2} d x^{3}\right)+2 e d x^{2} d x^{4}+e d x^{3^{2}}+\sum_{\sigma=5}^{n} e_{\sigma} \lambda_{\sigma} d x^{\sigma^{2}},
\end{aligned}
$$

где $\sigma=5,6, \ldots, n, e, e_{\sigma}= \pm 1, \lambda, \lambda_{\sigma}$ являются корнями характеристического уравнения $\operatorname{det}\left(h_{i j}-\lambda g_{i j}\right)=0$, причем $\lambda \neq \lambda_{\sigma}, \lambda_{\sigma} \neq \lambda_{\tau}$ при $\sigma \neq \tau$. 


\section{2. МЕТРИКА $h$-ПРОСТРАНСТВА ТИПА $[41 \ldots 1]$}

Подставляя канонические формы $\bar{g}_{p r}$ и $\bar{a}_{p q}$ в уравнение (4) и учитывая, что $e_{1}=$ $e_{2}=e_{3}=e_{4}=e$ (см. статью [7]), получаем следующую систему уравнений:

$$
\begin{gathered}
X_{r} \lambda=0 \quad(r \neq 4), \quad X_{r} \lambda_{\sigma}=0 \quad(r \neq \sigma), \quad X_{4}(2 \lambda-\phi)=X_{\sigma}\left(\lambda_{\sigma}-2 \phi\right)=0, \\
\gamma_{314}=\frac{1}{2} e X_{4} \phi, \quad \gamma_{413}=\gamma_{431}=\gamma_{422}=-e X_{4} \phi \\
\gamma_{1 \sigma 4}=\gamma_{2 \sigma 3}=\gamma_{3 \sigma 2}=\gamma_{4 \sigma 1}=\frac{e X_{\sigma} \phi}{\lambda-\lambda_{\sigma}}, \quad \gamma_{2 \sigma 4}=\gamma_{3 \sigma 3}=\gamma_{4 \sigma 2}=-\frac{e X_{\sigma} \phi}{\left(\lambda-\lambda_{\sigma}\right)^{2}} \\
\gamma_{3 \sigma 4}=\gamma_{4 \sigma 3}=\frac{e X_{\sigma} \phi}{\left(\lambda-\lambda_{\sigma}\right)^{3}}, \quad \gamma_{4 \sigma 4}=-\frac{e X_{\sigma} \phi}{\left(\lambda-\lambda_{\sigma}\right)^{4}} \\
\gamma_{4 \sigma \sigma}=\frac{e_{\sigma} X_{4} \phi}{\lambda-\lambda_{\sigma}}, \quad \gamma_{\sigma \tau \sigma}=\frac{e_{\sigma} X_{\tau} \phi}{\lambda_{\sigma}-\lambda_{\tau}}
\end{gathered}
$$

где $\sigma, \tau=5,6, \ldots, n, \sigma \neq \tau$, остальные инварианты $\gamma_{p q r}$ равны нулю. Для того чтобы система линейных дифференциальных уравнений в частных производных

$$
X_{q} \theta=\xi_{q}^{i} \partial_{i} \theta=0, \quad q=1, \ldots, m, \quad i=1, \ldots, n, \quad m<n,
$$

где $\xi_{q}^{i}$ - компоненты косонормального репера, была вполне интегрируемой, т. е. чтобы она допускала $n-m$ независимых решений, необходимо и достаточно, чтобы все коммутаторы операторов системы (см. работу [2])

$$
\left[X_{q}, X_{r}\right]=X_{q} X_{r}-X_{r} X_{q}=\sum_{p=1}^{n} e_{p}\left(\gamma_{p q r}-\gamma_{p r q}\right) X_{\tilde{p}}
$$

линейно выражались через операторы $X_{q}$.

Используя формулы (6) и (8), выпишем коммутаторы операторов $X_{i}, i=1, \ldots, n$, в рассматриваемом $h$-пространстве:

$$
\begin{gathered}
\left(X_{1}, X_{2}\right)=0, \quad\left(X_{1}, X_{3}\right)=0, \quad\left(X_{2}, X_{3}\right)=0, \quad\left(X_{1}, X_{4}\right)=e\left(\gamma_{314}-\gamma_{341}\right) X_{2}, \\
\left(X_{2}, X_{4}\right)=-e \gamma_{242} X_{3}, \quad\left(X_{3}, X_{4}\right)=e\left(\gamma_{134}-\gamma_{143}\right) X_{4}, \\
\left(X_{1}, X_{\sigma}\right)=-e \gamma_{4 \sigma 1} X_{1}, \quad\left(X_{2}, X_{\sigma}\right)=-e \gamma_{4 \sigma 2} X_{1}-e \gamma_{3 \sigma 2} X_{2}, \\
\left(X_{3}, X_{\sigma}\right)=-e \gamma_{4 \sigma 3} X_{1}-e \gamma_{3 \sigma 3} X_{2}-e \gamma_{2 \sigma 3} X_{3}, \\
\left.X_{4}, X_{\sigma}\right)=-e \gamma_{4 \sigma 4} X_{1}-e \gamma_{3 \sigma 4} X_{2}-e \gamma_{2 \sigma 4} X_{3}-e \gamma_{1 \sigma 4} X_{4}+e \gamma_{\sigma 4 \sigma} X_{\sigma}, \\
\left(X_{\sigma}, X_{\tau}\right)=-e \gamma_{\sigma} \gamma_{\sigma \tau \sigma} X_{\sigma}+e \gamma_{\tau \sigma \tau} X_{\tau} .
\end{gathered}
$$

Далее, составляя вполне интегрируемые системы (7) из (9), мы определяем допускаемые этими системами независимые решения, которые обозначим через $\theta^{i}$. После чего с помощью преобразования координат $x^{i^{\prime}}=\theta^{i}(x)$ мы можем обратить в нуль некоторые компоненты $\xi^{i}$ введенного выше косонормального репера. В частности, вполне интегрируемыми системами являются системы $X_{p} \theta=0(p \neq 4), X_{q} \theta^{\sigma}=0$ $(q \neq \sigma), X_{1} \theta=X_{\sigma} \theta=0, X_{1} \theta=X_{2} \theta=X_{\sigma} \theta=0$ и $X_{\sigma} \theta=0$. Легко заметить, что первая система имеет решение $\theta^{4}$, вторая система - решение $\theta^{\sigma}$, третья система решения $\theta^{2}, \theta^{3}, \theta^{4}$, четвертая $-\theta^{3}, \theta^{4}$, пятая $-\theta^{1}, \theta^{2}, \theta^{3}$ и $\theta^{4}$. 
После преобразования координат $x^{i^{\prime}}=\theta^{i}(x)$ в новой системе координат, опуская штрихи, находим

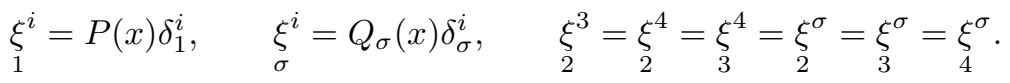

С помощью полученных равенств можно легко проинтегрировать уравнения (6), не содержащие $\gamma_{p q r}$, и найти функцию $\varphi$. Она имеет вид

$$
2 \varphi=\sum_{i=1}^{n} f_{i}+c, \quad \lambda_{i}=f_{i},
$$

где $\lambda_{1}=\lambda_{2}=\lambda_{3}=\lambda_{4}=\lambda, f_{1}=f_{2}=f_{3}=f_{4}\left(x^{4}\right), f_{\sigma}\left(x^{\sigma}\right)-$ произвольные функции указанных переменных, $c=$ const.

Приравнивая коэффициенты при одинаковых производных $\partial / \partial x^{i}$ в правых и левых частях равенств (9), с помощью формул $(6),(10)$ и (11) получаем довольно громоздкую систему дифференциальных уравнений в частных производных на компоненты $\underset{q}{\xi^{i}}$ косонормального репера. Интегрируя эту систему уравнений с удачно подобранными преобразованиями координат (подобные выкладки можно найти в статье [11]), а затем применяя формулы (см. работу [7])

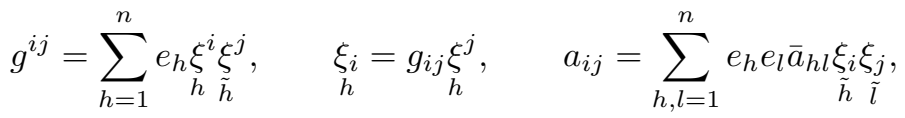

мы в конечном итоге находим компоненты тензоров $g_{i j}$ и $a_{i j}$.

Запишем итоговый результат в виде следующей теоремы.

ТЕОРема 1. Если симметрический тензор $h_{i j}$ типа [41...1] и скаляр ч удовлетворяют в $V^{n}\left(g_{i j}\right)$ уравнениям (3), то существует голономная система координат, в которой $\varphi, g_{i j}$ и $h_{i j}$ определяются формулами

$$
\begin{aligned}
& g_{i j} d x^{i} d x^{j}=e \Pi_{\sigma}\left(f_{\sigma}-\epsilon x^{4}\right)\left(6 A d x^{1} d x^{4}+2 d x^{2} d x^{3}+\right. \\
&+ 2\left(2 \epsilon x^{2}-3 A \Sigma\right) d x^{2} d x^{4}-\Sigma\left(d x^{3}\right)^{2}+2\left(\epsilon x^{1}-2 \epsilon x^{2} \Sigma\right) d x^{3} d x^{4}+ \\
&+\left.4\left(\left(\epsilon x^{2}\right)^{2} \Sigma+\epsilon^{2} x^{1} x^{2}-\frac{3}{2} \epsilon x^{1} A \Sigma\right)\left(d x^{4}\right)^{2}\right)+ \\
&+ 3 A d x^{3} d x^{4}+12 \epsilon x^{2} A\left(d x^{4}\right)^{2}+\sum_{\sigma} e_{\sigma} \Pi_{i}^{\prime}\left(f_{i}-f_{\sigma}\right)\left(d x^{\sigma}\right)^{2}, \\
& a_{i j} d x^{i} d x^{j}=\epsilon x^{4} g_{i_{1} j_{1}} d x^{i_{1}} d x^{j_{1}}+2 g_{14} d x^{2} d x^{4}+2 g_{24} d x^{3} d x^{4}+ \\
&+g_{23}\left(d x^{3}\right)^{2}+3 g_{23}\left(2 \epsilon x^{1} A-4 \epsilon x^{2} A \Sigma+\frac{4}{3}\left(\epsilon x^{2}\right)^{2}\right)\left(d x^{4}\right)^{2}+ \\
&+9 A^{2}\left(d x^{4}\right)^{2}+\sum_{\sigma} f_{\sigma} g_{\sigma \sigma}\left(d x^{\sigma}\right)^{2}, \\
& h_{i j}=a_{i j}+2 \varphi g_{i j}, \quad 2 \varphi=4 \epsilon x^{4}+\sum_{\sigma} f_{\sigma}+c \\
& A=\epsilon x^{3}+\theta\left(x^{4}\right), \quad \Sigma=\sum_{\sigma}\left(f_{\sigma}-\epsilon x^{4}\right)^{-1},
\end{aligned}
$$


где $i=1,2, \ldots, n, \sigma=5,6, \ldots, n, i_{1}, j_{1}=1, \ldots, 4, e, e_{\sigma}= \pm 1, f_{1}=f_{2}=f_{3}=f_{4}=\epsilon x^{4}$, $f_{\sigma}$ - функиия переменной $x^{\sigma}, f_{4}, f_{\sigma}$ - не равные тождественно друг другу функиии, $\epsilon$ равно нулю или единице, $\theta$ - функция переменной $x^{4}$, отличная от нуля при $\epsilon=0$, $\Pi_{\sigma}$ означает произведение по $\sigma, \Pi_{i}^{\prime}$ означает произведение для всех $i=1, \ldots, n$, кроме $i=\sigma$.

В данной теореме сформулированы необходимые условия. Непосредственной проверкой можно убедиться в том, что справедливо и обратное утверждение, поэтому найденные условия являются достаточными. Таким образом, имеет место следующее утверждение.

Теорема 2. Для того чтобы псевдориманово пространство $V^{n}\left(g_{i j}\right)$ было $h$-пространством типа [41 ...1], необходимо и достаточно, чтобы в некоторой голономной системе координат его метрика определялась формулой (12).

Напомним, что проективное движение $\xi$ является проективным движением типа $\chi$, если производная Ли $L_{\xi} g_{i j}$ метрического тензора $g_{i j}$ принадлежит к типу $\chi$ (см. статью [7]). В нашем случае $\chi=[41 \ldots 1]$. Движение $\xi$ есть проективное движение в $V^{n}\left(g_{i j}\right)$ тогда и только тогда, когда выполняются обобщенные уравнения Киллинга $L_{\xi} g_{i j}=h_{i j}$. Поэтому справедлива следующая теорема.

Теорема 3. Для того чтобы бесконечно малое преобразование $x^{i^{\prime}} \rightarrow x^{i}+\xi^{i} \delta t$ было проективным движением типа [41...1] в п-мерном псевдоримановом пространстве $V^{n}\left(g_{i j}\right)$, необходимо и достаточно, чтобы в некоторой системе координат

$$
d s^{2}=g_{i j} d x^{i} d x^{j}, \quad L_{\xi} g_{i j}=h_{i j},
$$

где соответствующие пары $g_{i j} u h_{i j}$, а также отвечающая им определяющая бункиия $\varphi$ определень в теореме 1.

\section{3. УСЛОВИЯ ПОСТОЯНСТВА КРИВИЗНЫ $h$-ПРОСТРАНСТВА ТИПА $[41 \ldots 1]$}

Для дальнейшего исследования нам понадобятся необходимые и достаточные условия постоянства кривизны рассматриваемого $h$-пространства.

Как известно, это условие выражается равенством

$$
R_{j k l}^{i}=K\left(\delta_{k}^{i} g_{j l}-\delta_{l}^{i} g_{j k}\right), \quad K=\text { const. }
$$

Отличные от нуля компоненты тензора кривизны заданного $h$-пространства имеют вид $R_{\nu i \mu}^{i}, i \neq \mu, R_{\nu b_{1} \mu}^{a_{1}}, b_{1} \neq \mu, a_{1}<b_{1}$. Здесь $i=1,2, \ldots, n, a_{1}, b_{1}=1, \ldots, 4, \nu, \mu$ являются индексами ненулевых компонент метрики $g_{\nu \mu}(12)$.

Из формулы (13) в частности получаем, что

$$
R_{114}^{1}=R_{1 \sigma 4}^{\sigma}, \quad R_{214}^{1}=R_{2 \sigma 4}^{\sigma}, \quad R_{224}^{1}=0 .
$$

Для $h$-пространства типа $[41 \ldots 1]$

$$
\begin{gathered}
R_{114}^{1}=\rho g_{14}, \quad R_{214}^{1}=\rho g_{24}, \quad R_{1 \sigma 4}^{\sigma}=\rho_{\sigma} g_{14}, \quad R_{2 \sigma 4}^{\sigma}=\rho_{\sigma} g_{24} \\
R_{224}^{1}=\gamma_{1} g_{24}+\gamma_{2} g_{14}+\frac{2 \epsilon}{3 A^{2}}\left(\theta^{\prime}\left(x^{4}\right)-\frac{4}{3} \epsilon^{2} x^{2}\right)
\end{gathered}
$$


где

$$
\begin{aligned}
& \gamma_{1}=- \frac{1}{4} \sum_{\sigma} \frac{\left(f_{\sigma}^{\prime}\right)^{2}}{\left(f_{\sigma}-\epsilon x^{4}\right)^{3} g_{\sigma \sigma}}, \quad \gamma_{2}=-\frac{1}{4} \sum_{\sigma} \frac{\left(f_{\sigma}^{\prime}\right)^{2}}{\left(f_{\sigma}-\epsilon x^{4}\right)^{4} g_{\sigma \sigma}}, \\
& \rho=-\frac{1}{4} \sum_{\sigma} \frac{\left(f_{\sigma}^{\prime}\right)^{2}}{\left(f_{\sigma}-\epsilon x^{4}\right)^{2} g_{\sigma \sigma}}, \\
& \rho_{\sigma}=-\frac{1}{4} \frac{\left(f_{\sigma}^{\prime}\right)^{2}}{\left(f_{\sigma}-\epsilon x^{4}\right) g_{\sigma \sigma}}\left(\frac{2 f_{\sigma}^{\prime \prime}}{\left(f_{\sigma}^{\prime}\right)^{2}}-\frac{1}{f_{\sigma}-\epsilon x^{4}}+\sum_{i, i \neq \sigma}\left(f_{i}-f_{\sigma}\right)^{-1}\right)- \\
&-\frac{1}{4} \sum_{\gamma, \gamma \neq \sigma} \frac{\left(f_{\gamma}^{\prime}\right)^{2}}{\left(f_{\gamma}-\epsilon x^{4}\right)\left(f_{\gamma}-f_{\sigma}\right) g_{\gamma \gamma}},
\end{aligned}
$$

а индексы $\sigma, \gamma=5,6, \ldots, n$.

Следовательно, из первых двух равенств (14) находим, что

$$
\rho-\rho_{\sigma}=0, \quad \gamma_{1} g_{14}+\frac{2 \epsilon^{2}}{3 A}=0 .
$$

Дифференцируя последнее соотношение по $x^{3}$, получаем равенство $\epsilon=0$, откуда $\gamma_{1}=0$. Тогда из последнего равенства (14) следует, что $\gamma_{2}=0$.

Покажем, что необходимые условия постоянства кривизны $h$-пространства типа $[41 \ldots 1]$

$$
\rho-\rho_{\sigma}=\epsilon=\gamma_{1}=\gamma_{2}=0
$$

являются достаточными. Имеем

$$
\begin{gathered}
\partial_{1} \rho=\partial_{2} \rho=\partial_{3} \rho=0 \\
\partial_{4} \rho=6 \epsilon \gamma_{1}, \quad \partial_{\sigma} \rho=-f_{\sigma}^{\prime} \frac{\rho-\rho_{\sigma}}{f_{\sigma}-\epsilon x^{4}} .
\end{gathered}
$$

Отсюда в силу условия (15) получаем, что $\rho=\rho_{\sigma}=$ const. Нетрудно убедиться, что при выполнении последнего равенства и условий $\epsilon=\gamma_{1}=\gamma_{2}=0$ тензор кривизны рассматриваемого пространства удовлетворяет соотношениям (13) с $K=\rho=$ const.

Сформулируем результат, полученный в настоящем разделе, в виде следующей теоремы.

Теорема 4. Для того чтобъ h-пространство $V^{n}\left(g_{i j}\right)$ muпа $[41 \ldots 1]$ имело постоянную кривизну, необходимо и достаточно выполнение условий (15).

\section{4. ОБ ОПРЕДЕЛЯЮЩЕЙ ФУНКЦИИ ПРОЕКТИВНОГО ДВИЖЕНИЯ В $h$-ПРОСТРАНСТВЕ ТИПА [41..1]}

Здесь доказана следующая теорема.

Теорема 5. Всякая определяющая функиия проективного движения в $h$-пространстве непостоянной кривизнъ типа [41 ...1] есть $\phi=a_{1} \varphi$, где $a_{1}=$ const, $\varphi-$ функция, определенная в теореме 1. 
ДокАЗАтЕльство. Пусть векторное поле $\xi^{i}$ задает проективное движение с определяющей функцией $\phi$ в рассматриваемом $h$-пространстве $V^{n}\left(g_{i j}\right)$. Тогда для тензора $b_{i j}=\underset{\xi}{L} g_{i j}$ выполняются уравнения Эйзенхарта

$$
b_{i j, k}=2 g_{i j} \phi_{, k}+g_{i k} \phi_{, j}+g_{j k} \phi_{, i} .
$$

Условия их интегрируемости имеют вид

$$
b_{m i} R_{j k l}^{m}+b_{m j} R_{i k l}^{m}=g_{i k} \phi_{, j l}+g_{j k} \phi_{, i l}-g_{l i} \phi_{, j k}-g_{l j} \phi_{, i k} .
$$

Покажем, что для любого проективного движения в рассматриваемом $h$-пространстве выполняются условия

$$
b_{\alpha \beta}=0, \quad \phi_{, \alpha \beta}=0,
$$

где $\alpha, \beta$ являются индексами нулевых компонент $g_{\alpha \beta}$ соответствующего метрического тензора.

$$
\begin{gathered}
\text { При }(i j k l)=(1213),(1313),(1 \sigma 1 \sigma),(3312),(1314) \text { получаем равенства } \\
b_{11} R_{213}^{1}=-g_{23} \phi_{, 11}, \quad b_{11} R_{313}^{1}=-g_{33} \phi_{, 11}, \\
b_{11} R_{\sigma 1 \sigma}^{1}=-g_{\sigma \sigma} \phi_{, 11}, \\
b_{13} R_{312}^{1}=-g_{23} \phi_{, 13}, \quad b_{11} R_{314}^{1}+b_{13} R_{114}^{1}=-g_{14} \phi_{, 13}-g_{34} \phi_{, 11} .
\end{gathered}
$$

Так как

$$
\begin{gathered}
R_{213}^{1}=R_{312}^{1}=g_{23} \rho, \quad R_{114}^{1}=g_{14} \rho \\
R_{313}^{1}=g_{33} \rho+\gamma_{1} g_{23}+\frac{2 \epsilon^{2}}{9 A^{2}}, \quad R_{\sigma 1 \sigma}^{1}=g_{\sigma \sigma} \rho_{\sigma} \\
R_{314}^{1}=g_{34} \rho+\gamma_{1} g_{24}+\gamma_{2} g_{14}+\frac{2 \epsilon}{3 A^{2}}\left(\theta^{\prime}-\frac{4 \epsilon^{2} x^{2}}{3}\right),
\end{gathered}
$$

то из уравнений (19) и (20) вытекает, что

$$
b_{11}\left(\rho-\rho_{\sigma}\right)=b_{11}\left(\rho-\rho_{\sigma}+\gamma_{1} \frac{g_{23}}{g_{33}}+\frac{2 \epsilon^{2}}{9 A^{2} g_{33}}\right)=0 .
$$

Из уравнений $(20)$ и (21) следует, что

$$
b_{11}\left(\rho-\rho_{\sigma}+\gamma_{1} \frac{g_{24}}{g_{34}}+\gamma_{2} \frac{g_{14}}{g_{34}}+\frac{2 \epsilon}{3 A^{2} g_{34}}\left(\theta^{\prime}-\frac{4 \epsilon^{2} x^{2}}{3}\right)\right)=0 .
$$

Предположим, что $b_{11} \neq 0$. Тогда из последних соотношений легко получить равенства $\rho-\rho_{\sigma}=\epsilon=\gamma_{1}=\gamma_{2}=0$, которые согласно теореме 4 являются необходимыми и достаточными условиями постоянства кривизны, т. е. рассматриваемое пространство имеет постоянную кривизну, что противоречит доказываемой теореме. Таким образом, $b_{11}=0$ и, следовательно, $\phi_{, 11}=0$. Подобным образом доказываются остальные соотношения (18).

С помощью доказанных соотношений (18) из уравнений (16), полагая поочередно $(i j k)=(1 \tau \tau),(2 \tau \tau),(3 \tau \tau), \tau=5,6, \ldots, n$, получаем соотношения

$$
\phi_{, 1}=\phi_{, 2}=\phi_{, 3}=0,
$$




$$
\phi_{, \sigma}=f_{\sigma}^{\prime} P_{\sigma \tau}, \quad \phi_{, \tau}=f_{\tau}^{\prime} P_{\sigma \tau}, \quad P_{\sigma \tau} \equiv \frac{1}{2} \frac{b_{\tau \tau} g_{\tau \tau}^{-1}-b_{\sigma \sigma} g_{\sigma \sigma}^{-1}}{f_{\tau}-f_{\sigma}}, \quad \sigma \neq \tau
$$

Из последних равенств следует равенство $\partial_{\sigma \tau} \phi=0, \sigma, \tau=5,6, \ldots, n, \sigma \neq \tau$. Используя полученные результаты, из уравнений $(16)$ при $(i j k)=(1 \tau 4),(4 \tau \tau),(\tau \tau \tau)$ находим

$$
\begin{gathered}
\phi_{, \tau}=\frac{1}{2} \frac{f_{\tau}^{\prime}}{f_{\tau}-\epsilon x^{4}}\left(\frac{b_{\tau \tau}}{g_{\tau \tau}}-\frac{b_{14}}{g_{14}}\right), \quad \phi_{, 4}=2 \frac{\epsilon}{f_{\tau}-\epsilon x^{4}}\left(\frac{b_{\tau \tau}}{g_{\tau \tau}}-\frac{b_{14}}{g_{14}}\right), \\
\frac{\partial_{\tau} b_{\tau \tau}}{g_{\tau \tau}}=-f_{\tau}^{\prime} \sum_{i, j \neq \tau}\left(f_{i}-f_{\tau}\right)^{-1} \frac{b_{\tau \tau}}{g_{\tau \tau}}+4 \phi_{, \tau}, \quad i, j=1,2, \ldots, n,
\end{gathered}
$$

откуда несложно вывести уравнения

$$
f_{\tau}^{\prime} \partial_{\tau} \phi_{, \tau}=f_{\tau}^{\prime \prime} \phi_{, \tau}, \quad 4 \epsilon \phi_{, \tau}=f_{\tau}^{\prime} \phi_{, 4}
$$

Из условия $\phi_{, 4 \tau}=0$ и соотношения $4 \epsilon \phi_{, \tau}=f_{\tau}^{\prime} \phi_{, 4}$ следует равенство $\partial_{4 \tau} \phi=0$. Если не все $f_{\tau}^{\prime}=0$, то из полученных формул вытекает нужный результат

$$
\phi=\frac{1}{2} a_{1} \sum_{i=1}^{n} f_{i}=a_{1} \varphi
$$

где $\varphi$ определяется формулой (11). В противном случае, когда все $f_{\tau}^{\prime}=0$, имеем

$$
\begin{gathered}
\phi_{, \tau}=\phi_{, 14}=\phi_{, 23}=\phi_{, 24}=\phi_{, \tau \tau}=0, \quad \phi_{, 34}=-\frac{2 \epsilon^{2}}{3 A\left(f_{\tau}-\epsilon x^{4}\right)}\left(\frac{b_{\tau \tau}}{g_{\tau \tau}}-\frac{b_{14}}{g_{14}}\right), \\
\phi_{, 44}=\partial_{44} \phi-\frac{2 \epsilon\left(3 \theta^{\prime}-2 \epsilon^{2} x^{2}\right)}{3 A\left(f_{\tau}-\epsilon x^{4}\right)}\left(\frac{b_{\tau \tau}}{g_{\tau \tau}}-\frac{b_{14}}{g_{14}}\right)
\end{gathered}
$$

где $\epsilon=1$, так как при $\epsilon=f_{\tau}^{\prime}=0$ пространство имеет нулевую кривизну.

Из уравнений $(17)$ при $(i j k l)=(4413),(4 \tau 4 \tau)$ получаем два равенства

$$
\begin{aligned}
b_{14} R_{413}^{1}+b_{24} R_{413}^{2} & =g_{14} \phi_{, 34}, \\
b_{14} R_{\tau 4 \tau}^{1}+b_{24} R_{\tau 4 \tau}^{2}+b_{\tau \tau} R_{44 \tau}^{\tau} & =-g_{\tau \tau} \phi_{, 44} .
\end{aligned}
$$

Выражая $b_{24}$ из первого равенства и подставляя результат во второе равенство, с учетом формулы (22) получаем равенство $\partial_{44} \phi=0$, откуда и следует нужный результат. Итак, теорема доказана.

\section{5. О КОВАРИАНТНО ПОСТОЯННОМ СИММЕТРИЧЕСКОМ ТЕНЗОРЕ В $h$-ПРОСТРАНСТВЕ ТИПА [41...1]}

Докажем следующую теорему.

ТЕОРема 6. Любой ковариантно постоянный симметрический тензор $b_{i j}$ в $h$ пространстве непостоянной кривизны типа [41..1] пропорционален фундаментальному тензору:

$$
b_{i j}=a_{2} g_{i j}, \quad a_{2}=\text { const. }
$$


ДокАЗАтельство. Пусть $b_{i j}$ удовлетворяет в $V^{n}\left(g_{i j}\right)$ уравнениям

$$
b_{i j, k}=0,
$$

которые получаются из уравнений (16) при $\phi=$ const. Следовательно, условия интегрируемости уравнений (24) определяются равенствами (17) при $\phi=$ const. Тогда из условий (18) получаем равенство $b_{\alpha \beta}=0$. Напомним, что индексы $\alpha, \beta$ соответствуют индексам нулевых компонент метрического тензора $g_{\alpha \beta}$ рассматриваемого $h$-пространства. Для доказательства теоремы достаточно показать, что $b_{i j}=a_{2} g_{i j}$, где $a_{2}$ - некоторый коэффициент пропорциональности, постоянство которого следует из уравнений $b_{i j, k}=0$ и параллельности метрического тензора [7].

Из уравнения $(24)$ с $(i j k)=(\mu \tau \nu),(\sigma \tau \sigma), \sigma, \tau=5,6, \ldots, n, \sigma \neq \tau$, получаем соотношения

$$
f_{\tau}^{\prime}\left(\frac{b_{\tau \tau}}{g_{\tau \tau}}-\frac{b_{\mu \nu}}{g_{\mu \nu}}\right)=0,
$$

где $\mu, \nu$ являются индексами ненулевых компонент метрического тензора (12). Если не все $f_{\tau}^{\prime}=0$, то выполняется равенство

$$
\frac{b_{\mu \nu}}{g_{\mu \nu}}=\frac{b_{\tau \tau}}{g_{\tau \tau}}
$$

которое вместе с условием $b_{\alpha \beta}=0$ дает нужный результат $(23)$.

В случае $f_{\tau}^{\prime}=0$ последнее равенство следует из уравнений $(24)$ с $(i j k)=(\tau 4 \tau)$ и из $(17)$ с $(i j k l)=(3324),(4413),(2343),(4432),(4442)$ :

$$
\begin{gathered}
\epsilon\left(\frac{b_{\tau \tau}}{g_{\tau \tau}}-\frac{b_{14}}{g_{14}}\right)=0, \\
b_{23} R_{324}^{2}-b_{33} R_{342}^{3}=0, \quad b_{14} R_{413}^{1}-b_{24} R_{431}^{2}=0, \\
b_{23} R_{343}^{3}+b_{24} R_{343}^{4}+b_{23} R_{243}^{2}=0, \quad b_{14} R_{432}^{1}+b_{34} R_{432}^{3}=0, \\
b_{14} R_{442}^{1}+b_{24} R_{442}^{2}+b_{34} R_{442}^{3}+b_{44} R_{442}^{4}=0,
\end{gathered}
$$

где $\epsilon=1$ в силу непостоянства кривизны пространства, а компоненты тензора кривизны имеют вид

$$
\begin{gathered}
R_{431}^{2}=R_{342}^{3}=R_{432}^{3}=3 A R_{343}^{4}=\frac{R_{342}^{2}}{\Sigma}=\frac{R_{243}^{2}}{\Sigma}=\frac{2 \epsilon^{2}}{3 A}, \\
R_{343}^{3}=-\frac{4 \epsilon^{3} x^{2}}{9 A^{2}}, \quad R_{424}^{2}=3 A R_{423}^{1}=\frac{2 \epsilon^{2}}{3 A}\left(2 \epsilon x^{1}-2 \epsilon x^{2} \Sigma+3 A \Sigma_{1}\right), \\
R_{442}^{1}=-\frac{2 \epsilon \theta^{\prime} g_{34}}{3 A^{2} g_{23}}+\frac{4 \epsilon}{3 A}\left(\epsilon^{2} x^{2} \Sigma_{1}+\epsilon^{2} x^{2} \Sigma^{2}+\frac{2 \epsilon}{3 A} \epsilon^{2} x^{1} x^{2}-\frac{4 \epsilon}{3 A}\left(\epsilon x^{2}\right)^{2} \Sigma+\frac{3 A \epsilon \Sigma}{g_{23}}\right), \\
\Sigma_{1}=\sum_{\sigma}\left(f_{\sigma}-\epsilon x^{4}\right)^{-4} .
\end{gathered}
$$

Таким образом, теорема доказана.

\section{6. ПРОЕКТИВНО-ГРУППОВЫЕ СВОЙСТВА $h$-ПРОСТРАНСТВА $V^{N}\left(g_{i j}\right)$ ТИПА [41...1]}

Нетрудно заметить, что прямым следствием теоремы, доказанной в предыдущем разделе, является следующая теорема. 
ТЕорема 7. Афбинная группа в $h$-пространстве $V^{n}\left(g_{i j}\right)$ muпа $[41 \ldots 1]$ непостоянной кривизны состоит из гомотетий.

Общее решение уравнений Эйзенхарта в рассматриваемом $h$-пространстве непостоянной кривизны имеет вид $a_{1} h_{i j}+a_{2} g_{i j}$, где $a_{1}, a_{2}$ - произвольные постоянные,

$$
h_{i j}=a_{i j}+\left(\sum_{k=1}^{n} f_{k}\right) g_{i j}
$$

тензоры $a_{i j}, g_{i j}$ определены в теореме 1 . Это следует из теорем, доказанных в предыдущих разделах, линейности уравнения Эйзенхарта (3) и того факта, что любые два решения $\stackrel{1}{h}_{i j}$ и $\stackrel{2}{h}_{i j}$ этого уравнения с одинаковой правой частью могут отличаться лишь на ковариантно постоянный тензор $b_{i j}[7]$.

Таким образом, получаем следующую теорему.

Теорема 8. Все проективные движения в $h$-пространстве $V^{n}\left(g_{i j}\right)$ типа [41 ...1] непостоянной кривизны получаются интегрированием уравнений

$$
L_{\xi} g_{i j}=\xi_{i, j}+\xi_{j, i}=a_{1} a_{i j}+\left(a_{1} \sum_{k=1}^{n} f_{k}+a_{2}\right) g_{i j}
$$

где тензоры $a_{i j}$ и $g_{i j}$ определены в теореме $1, a_{1}, a_{2}$ - постоянные.

Из этой теоремы вытекает важная групповая характеристика данного $h$-пространства.

Теорема 9. Если h-пространство $V^{n}\left(g_{i j}\right)$ muna $[41 \ldots 1]$ допускает негомотетическую проективную алгебру Ли $P_{r}$, то эта алгебра содержит подалгебру $H_{r-1}$ инфинитезималъных гомотетий размерности $r-1$.

\section{7. ЗАКЛЮЧЕНИЕ}

В настоящей работе исследованы псевдоримановы пространства с произвольной сигнатурой, допускающие негомотетические инфинитезимальные проективные преобразования. В частности, найдена метрика $h$-пространства типа [41 .. 1], а затем установлены важные проективно-групповые свойства этого $h$-пространства. Важно отметить, что полученные проективно-групповые свойства для $h$-пространства типа [41 ...1] справедливы и для пространств лоренцевой сигнатуры, а именно $h$-пространств типов [11 .. 1], [21 .. 1], [31 .. 1] (см. статью [7]), а также для шестимерных $h$-пространств типов [2211], [321], [33], [411], [51] (см. работу [8]). Таким образом, можно с уверенностью предположить, что данные проективно-групповые свойства имеют место для всех $n$-мерных $h$-пространств типов $\left[a_{1} a_{2} \ldots a_{m}\right]$ произвольной сигнатуры. Здесь числа $a_{1}, a_{2}, \ldots, a_{m}$ могут принимать любое из значений $1,2, \ldots$, причем $a_{1}+a_{2}+\cdots+a_{m}=n$.

Следует заметить, что остается открытой проблема восстановления векторного поля, определяющего инфинитезимальное проективное преобразование. Решение этой задачи сводится к интегрированию уравнения Киллинга. 


\section{Список литературы}

[1] Б. А. Дубровин, С.П. Новиков, А.Т. Фоменко, Современная геометрия, УРСС, М., 1998.

[2] Л.П. Эйзенхарт, Риманова геометрия, ИЛ, М., 1948.

[3] G. Konigs, "Appl. II": G. Darboux, Leçons sur la théorie généralle des surfaces. IV, Gauthier-Villars, Paris, 1896, 368-404.

[4] А. З. Петров, "О геодезическом отображении римановых пространств неопределенной метрики", Уч. зап. Казан. ун-та, 109:3 (1949), 7-36.

[5] G. Fubini, "Sui gruppi transformazioni geodetiche", Mem. Acc. Torino. Cl. Fif. Mat. Nat., 53 (1903), 261-313.

[6] А.С. Солодовников, "Проективные преобразования римановых пространств", УМН, 11:4(70) (1956), 45-116.

[7] А.В. Аминова, “Алгебры Ли инфинитезимальных проективных преобразований лоренцевых многообразий”, УМН, 50:1(301) (1995), 69-142.

[8] З.Х. Закирова, Проективно-групповые свойства 6-мерных теорий типа Калуиъь-Клейна, Дисс. ... канд. физ.-матем. наук, КГУ, Казань, 2001.

[9] З. Х. Закирова, "Первые интегралы уравнений геодезических $h$-пространств типа [51]", Межвуз. темат. сб. науч. тр., Труды геометрического семинара, 23, Изд-во Казан. матем. общ-ва, Казань, 1997, 57-64.

[10] З.Х. Закирова, "Первые интегралы уравнений геодезических h-пространств типа [411]", Изв. вузов. Матем., 1999, № 9, 78-79.

[11] З. Х. Закирова, "Жесткие шестимерные $h$-пространства постоянной кривизны", ТМФ, 158:3 (2009), 347-354.

[12] З. Х. Закирова, "Метрики 6-мерных h-пространств типов [3(21)], [(32)1], [(321)]”, Kраткие сообщения по физике ФИАН, 38:9 (2011), 270-274.

[13] З. Х. Закирова, "О некоторых специальных решениях уравнения Эйзенхарта”, Уфимский матем. журн., 5:3 (2013), 41-53. 